\title{
原位生成的高价碘试剂在有机合成中的应用进展
}

\author{
杨 柳 ${ }^{a}$ 许国贺 ${ }^{a}$ 马晶军*, $a$ 杨 倩 ${ }^{b}$ 冯 安 ${ }^{a}$ 崔景港 ${ }^{a}$ \\ $\left({ }^{a}\right.$ 河北农业大学渤海校区理工学院 河北黄骅 061100) \\ $\left({ }^{b}\right.$ 河北农业大学动物医学院 河北保定 071000)
}

\begin{abstract}
摘要 原位生成的高价碘试剂具有原子经济性、性能温和和绿色环保等优点, 在诸多合成和不对称催化等反应中表现 活跃. 详细介绍了原位生成高价碘的概念以及反应机理，根据不同的反应类型分别对原位生成的三价碘、五价碘以及 手性高价碘试剂在有机合成反应中的应用进行了归纳总结, 分析了原位生成的高价碘试剂目前面临的问题，并对今后 的发展趋势作了展望.
\end{abstract}

关键词 原位生成; 高价碘试剂; 合成反应

\section{Recent Advances in the Application of in-situ Generated Hypervalent lodine Reagents in Organic Synthesis}

\author{
Yang, Liu $^{a} \quad \mathrm{Xu}$, Guohe $^{a} \quad$ Ma, Jingjun ${ }^{*, a} \quad$ Yang, Qian $^{b} \quad{\text { Feng, } \text { An }^{a} \quad \text { Cui, Jinggang }}^{a}$ \\ ( ${ }^{a}$ College of Science \& Technoloy, Agricultural University of Hebei Bohai Campus, Huanghua, Hebei 061100) \\ $\left({ }^{b}\right.$ College of Veterinary Medicine, Hebei Agricultural University, Baoding, Hebei 071001)
}

\begin{abstract}
Based on the atomic economy, mild performance and environment friendly, the in-situ generation of hypervalent iodine reagents has been vastly applied in many significant oxidative reactions and asymmetric catalysis. In this paper, the progress of in-situ generated hypervalent iodine reagents is systematically reviewed, including conception and mechanisms. According to the different reaction types, the application of in-situ generated hapervalent iodine reagent in organic synthesis reaction is summarized, including trivalent iodine reagent, pentavalent iodine reagent and chiral hypervalent iodine reagent. The problem of in-situ generated hapervalent iodine researches is analyzed, and the future development of in-situ hapervalent iodine is prospected.

Keywords in-situ generation; hypervalent iodine reagent; organic synthesis
\end{abstract}

高价碘试剂被称为超价碘试剂, 这是一类特殊的含 有碘元素的有机分子. 与一般的含碘化合物相比, 此类 分子中碘元素在形成化合物时, 自身的价电子总数超过 了八隅体规则所允许的数目, 此时碘显现为高价态 ${ }^{[1]}$. 一般高价碘可分为三价碘试剂或五价碘试剂. 高价碘试 剂本身是一种氧化剂, 其氧化能力与二价录 $(\mathrm{Hg}, \mathrm{II}) 、$ 四 价铅 $(\mathrm{Pb}, \mathrm{IV})$ 、六价铬 $(\mathrm{Cr}, \mathrm{VI})$ 和八价锇 $(\mathrm{Os}, \mathrm{VIII})$ 等金属 氧化剂相近, 但高选择性、性能温和等优点则是上述金 属氧化剂所不具备的 ${ }^{[2]} .19$ 世纪以来, 高价碘试剂在氧 化、加成、重排、取代以及不对称合成反应中都已有应 用 ${ }^{[3]}$, 作者曾利用三价碘试剂完成了四氢异喹啉中茮位
亚甲基的分子内官能团化 ${ }^{[4]}$. 随着社会对环保的要求越 来越高, 广泛使用的传统高价碘试剂显露了它的缺点, 如：(1)五价碘试剂具有潜在的爆炸性，不易保存，且价 格较昂贵; (2)很多氧化反应需要使用过量的高价碘试剂 参与反应，造成资源浪费; (3)传统高价碘试剂在反应中 主要被分解为芳基碘，不易回收再利用，且需要较复杂 的无害化后处理过程 ${ }^{[5]} ;(4)$ 高价碘试剂在有机溶剂中溶 解性不好, 限制了它们的使用范围. 这些问题成为设计 清洁、低成本的化学反应的瓶颈，限制了高价碘试剂在 工业领域中的应用 ${ }^{[6]}$.

因此，开发出既具有较高活性又能实现 “原子经济

\footnotetext{
* Corresponding author. E-mail: mjjwjpmartin@163.com

Received June 19, 2019; revised August 27, 2019; published online September 13, 2019.

Project supported by the Young Tip-Top Talents Plan of Universities and Colleges in Hebei Province (No. BJ201702), and the Specific Foundation for Doctor in Hebei Agriculture University of China (No. ZD2016027).

河北省高等学校青年拔尖人才计划(No. BJ201702)和河北农业大学引进博士专项(No. ZD2016027)资助项目.
} 


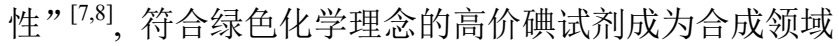
研究热点之一. 随着研究的深入, 人们发现原位生成的 高价碘不仅保留了高价碘试剂的所有优点, 而且催化剂 还可以被循环使用 ${ }^{[9]}$. 原位生成高价碘策略是在反应中 使用催化量的低价碘(碘为负一价)与等物质的量的氧化 剂反应，原位生成高价碘替代直接使用高价碘的一种合 成策略. 反应中常用催化剂有碘苯、取代碘苯、碘盐和单 质碘等碘化物, 氧化剂则包括间氯过氧苯甲酸 $(m \mathrm{CP}-$ BA)、过氧化氢 $\left(\mathrm{H}_{2} \mathrm{O}_{2}\right)$ 等过氧化物以及 $N$-溴代琥珀酰亚 胺(NBS)、过硫酸氢钾复合盐(Oxone)等. 详细介绍了原 位生成高价碘的机理, 反应类型, 以及近年来原位生成 的三价碘、五价碘以及手性高价碘试剂在有机合成反应 中的应用，并对原位生成高价碘试剂的发展做了展望.

\section{1 原位生成高价碘机理}

首先利用氧化剂将催化量的低价碘氧化为高价态 的碘, 生成的高价碘直接参与反应, 然后又释放出低价 碘, 如此反复循环. 在低价碘被氧化成高价碘的过程中, 不同的前催化剂产生的高价碘不同, 以下分别对芳基碘 和碘盐这两大类前催化剂原位生成高价碘的机理进行 阐述.

芳基碘作为前催化剂原位生成高价碘的机理见图 1. 价廉环保的过氧化物氧化剂 $\left(m C P B A 、 \mathrm{H}_{2} \mathrm{O}_{2}\right.$ 、过氧叔 丁醇 TBHP、过氧乙酸 $\mathrm{AcOOH}$ 等)先将催化量的碘苯氧 化成三价碘试剂, 后者参与反应将原料氧化成相应的产 物, 而后释放出的碘苯又进一步被氧化, 进而实现碘苯 的循环再利用. 另外反应生成的副产物安全可控, 如间 氯苯甲酸 $(m \mathrm{CBA})$ 、水、叔丁醇、乙酸等. 在催化量的低 价碘介导的循环体系中, 选择不同的氧化剂和催化剂可

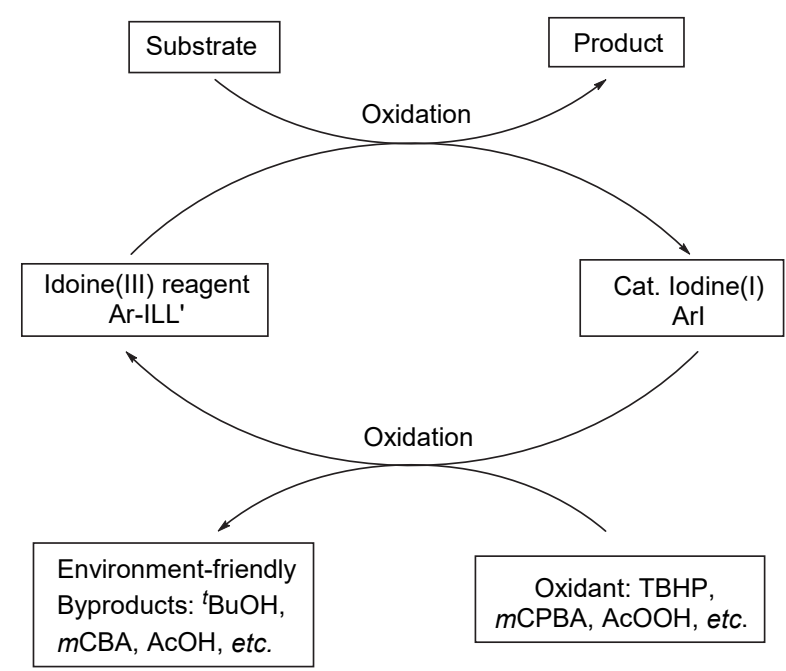

图 1 芳基碘作为前催化剂原位生成三价碘化合物示意图 Figure 1 Aryl iodide as a procatalyst in-situ generation of hypervalent iodine(III) reagent
分别得到三价碘或者五价碘中间体，低价态的碘氧化到 高价态的过程是该反应的关键步骤. 碘盐作为前催化剂 原位生成高价碘的机理如图 2. 过氧化物 $(m \mathrm{CPBA}$, $\mathrm{H}_{2} \mathrm{O}_{2}$, TBHP 等)将催化量的碘盐( $\mathrm{NaI}, \mathrm{KI}, \mathrm{Bu}_{4} \mathrm{NI}, \mathrm{Bu}_{4} \mathrm{PI}$ 等)氧化为正一价或正三价的高价碘 $\left(\mathrm{IO}^{-}\right.$或 $\left.\mathrm{IO}_{2}^{-}\right)$, 与图 1 过程类似, 后者参与氧化反应并释放出安全可控的副 产物 ${ }^{[10]}$

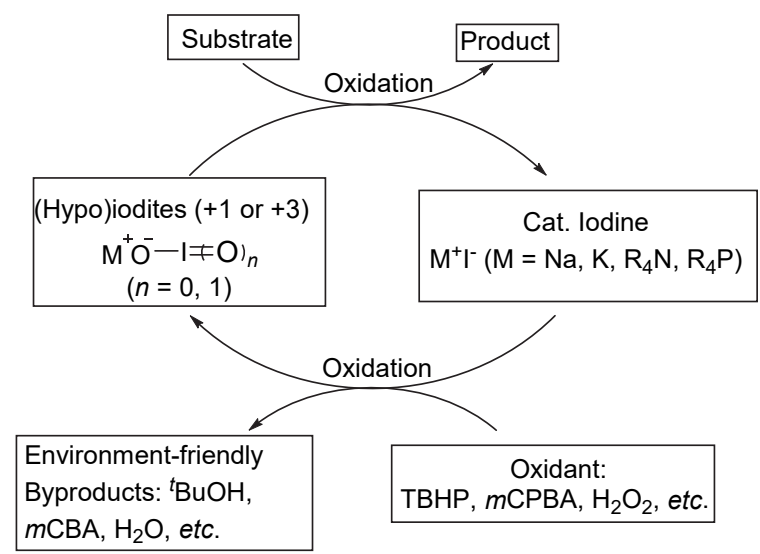

图 2 碘盐作为前催化剂原位生成高价碘化合物示意图 Figure 2 Iodide salts as precatalysts $i n$-situ generation of hypervalent iodine reagent

\section{2 原位生成三价碘试剂在合成中的应用}

\section{1 芳基磑试剂作为催化剂原位生成三价磑在合成中 的应用}

近年来原位生成三价碘试剂的催化循环反应已被 成功地应用到了杂环和螺环等化合物的构建中，其中芳 基碘试剂作为催化剂在催化循环反应中尤为常见. 总结 了芳基碘试剂作为催化剂，不同类型的氧化剂(如 $m \mathrm{CPBA}, \mathrm{AcOOH}, \mathrm{H}_{2} \mathrm{O}_{2}, \mathrm{TBHP}$ 等)原位生成三价碘在有 机合成中的应用.

2.1.1 $m$ CPBA 作为氧化剂原位生成三价碘在有机合 成中的应用

间氯过氧苯甲酸 $(m \mathrm{CPBA})$ 是一种过氧羧酸, 可被广 泛地应用于有机合成的氧化反应中. 主要应用领域有酮 转化为酯(Baeyer-Villiger 氧化)、烯烃环氧化(Prilezhaev 反应)和甲硅烷基烯醇醚转化为甲硅烷基 $\alpha$-差基酮 (Rubottom 氧化)等. 因 $m \mathrm{CPBA}$ 氧化性过强，易破坏原 料，降低收率，所以在原位生成反应过程中不直接用于 氧化原料, 而是使用氧化剂 $m \mathrm{CPBA}$ 将催化量的碘试剂 氧化为高价碘，后者作为氧化剂参与原料的氧化反应. 这类反应中可使用的氧化剂种类很多如 Oxone、 $\mathrm{H}_{2} \mathrm{O}_{2}$ 等, 因 $m \mathrm{CPBA}$ 氧化性强, 相对易处理, 所以应用较多. 但 是 $m \mathrm{CPBA}$ 在使用过程中要避免碰撞, 防止发生爆炸. 
以下对以 $m \mathrm{CPBA}$ 为氧化剂原位生成三价碘介导的去芳 构化反应、偶联反应、重排反应等作简要介绍.

2005 年, Ochiai 课题组 ${ }^{[11]}$ 首次运用原位生成三价碘 策略, 即以 0.1 equiv. 的碘苯作为催化剂, 2.0 equiv. $m \mathrm{CPBA}$ 作为氧化剂, 醋酸为原料, 在羰基化合物 $\mathbf{1}$ 的 $\alpha$ 位引入乙酰氧基生成目标化合物 2 (Eq. 1). 在反应过程 中 $m \mathrm{CPBA}$ 将催化量的碘苯氧化成三价碘二醋酸碘苯 (PIDA), PIDA 参与酮 1 的 $\alpha$ 位氧化, 生成的碘苯进一步 被氧化循环为三价碘. 该反应在室温条件下进行, 产率 中等( $43 \% \sim 63 \%$ ), 反应时间较长(超过 $20 \mathrm{~h}$ ). 在 2007 年, Togo 团队 ${ }^{[12]}$ 研究发现原位生成高价碘条件下, 用甲 磺酸替代醋酸同样可以实现酮类化合物 $\mathbf{3}$ 的 $\alpha$-对甲苯磺 酰氧基化，产率可达 85\%且时间缩短为 $5 \mathrm{~h}$ (Eq. 2). 同 时该课题组团队在原有基础上对醇类化合物 $\mathbf{5}$ 的 $\alpha$-甲苯 磺酰氧基化进行了研究, 研究表明, 通过 “一锅法”, 原 位生成的三价碘将醇氧化为酮, 随后实现酮类的甲磺酰 氧化(Scheme 1). 产物 $\alpha$-甲苯磺酸酮是重要的合成前体, 可以用于构建多种芳杂环化合物, 如噻唑、咪唑和啞唑 等.

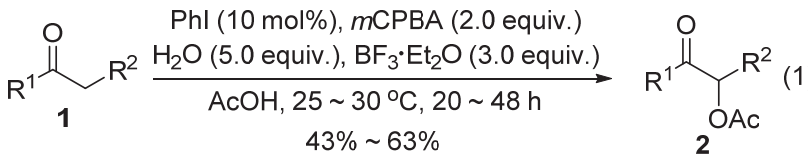

$$
\begin{aligned}
& \mathrm{R}^{1}={ }^{n} \mathrm{C}_{3} \mathrm{H}_{7},{ }^{n} \mathrm{C}_{4} \mathrm{H}_{9},{ }^{n} \mathrm{C}_{5} \mathrm{H}_{11}, \mathrm{Ph}, 4-\mathrm{FC}_{6} \mathrm{H}_{4} \text {, } \\
& \text { 4- } \mathrm{ClC}_{6} \mathrm{H}_{4}, 4-\mathrm{BrC}_{6} \mathrm{H}_{4}, 4-\mathrm{IC}_{6} \mathrm{H}_{4} \text {, etc. } \\
& \mathrm{R}^{2}=\mathrm{H}, \mathrm{Me}, \mathrm{Et},{ }^{n} \mathrm{C}_{3} \mathrm{H}_{7},{ }^{n} \mathrm{C}_{4} \mathrm{H}_{9}, \mathrm{CO}_{2} \mathrm{Et}
\end{aligned}
$$<smiles>CC(=O)c1ccccc1</smiles>

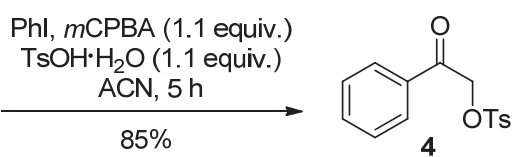

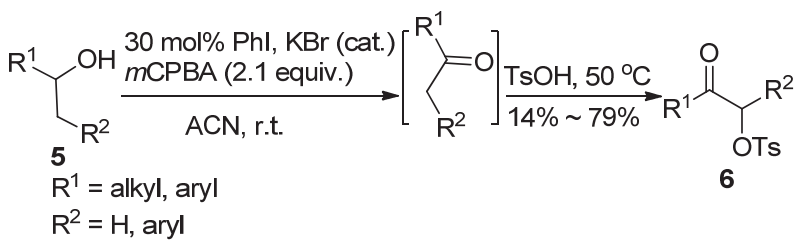

图式 $1 m \mathrm{CPBA}$ 作为氧化剂原位生成三价碘介导的羰基 $\alpha$ 位 官能团化

Scheme $1 m$ CPBA as an oxidant in situ to generate iodine(III) reagents-mediated oxidative functionalization at $\alpha$ positions of carbonyls

原位生成三价碘介导下的酚类去芳构化反应是合 成螺杂环化合物的重要方法之一, 可应用于呋喃衍生物 的骨架构建. 2005 年, Kita 等 ${ }^{[13]}$ 报道了原位生成三价碘 试剂介导下由酚类化合物 7 构建二氢吡喃酮型螺杂环化 合物 8 的反应. 该方法成功实现了原位生成高价碘介导
的酚类去芳构化，具有反应时间短、产率高等优点，最 高收率可达到 91\% (Eq. 3). 原位生成三价碘介导的去芳 构化还可以用于吡咯烷酮的构建. 2007 年, Kita 课题 组 ${ }^{[14]}$ 以 0.1 equiv. 的 4-甲基碘苯为催化剂, 1.5 equiv. 的 $m \mathrm{CPBA}$ 在室温条件下将原料酰胺类化合物 9 氧化完成, $\mathrm{C}-\mathrm{N}$ 键的构建, 合成了 $N$-稠合螺内酰胺 10 (Eq. 4).
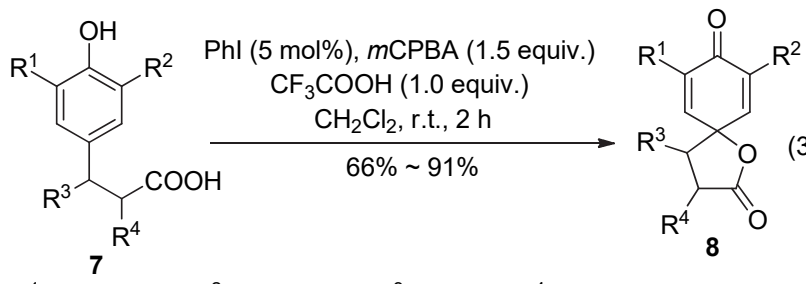

$\mathrm{R}^{1}=\mathrm{H}, \mathrm{Me}, \mathrm{Br} ; \mathrm{R}^{2}=\mathrm{H}, \mathrm{Me}, \mathrm{Br} ; \mathrm{R}^{3}=\mathrm{H}, \mathrm{Me} ; \mathrm{R}^{4}=\mathrm{H}, \mathrm{Me}$

$\mathrm{R}^{3}, \mathrm{R}^{4}=\mathrm{CH}-\mathrm{CH}=\mathrm{CH}-\mathrm{CH}=\left(\mathrm{R}^{3}\right.$ and $\mathrm{R}^{4}$ are linked to $\alpha, \beta$ carbons of the carboxyl group to form an aromatic ring)
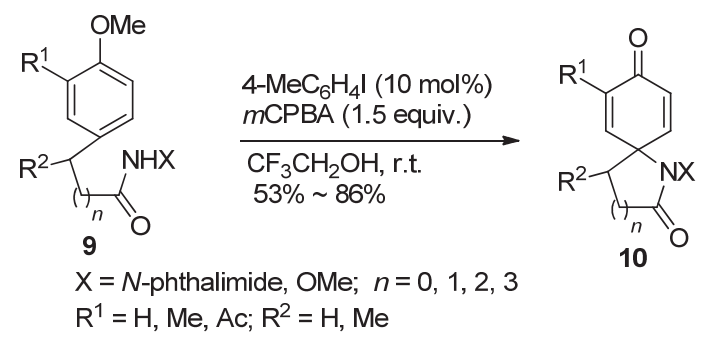

原位生成三价碘介导的氧化反应可以实现 $\mathrm{C}\left(\mathrm{sp}^{3}\right)$ $\mathrm{H}$ 和 $\mathrm{C}\left(\mathrm{sp}^{2}\right)-\mathrm{H}$ 的氨基化, 且产率较高. 2015 年, Shi 课 题组 ${ }^{[15]}$ 研究发现, 使用碘代芳烃和 $m \mathrm{CPBA}$ 组合在温和 条件下短时间可以完成分子内的叔 $\mathrm{C}-\mathrm{H}\left(\mathrm{sp}^{3}\right)$ 键的胺化. 通过密度泛函理论(M06-2X)计算表明，反应过程经历 了原位生成的三价碘介导的碘鎓阳离子中间体和 $\mathrm{C}-\mathrm{H}$ 活化/C-N键生成的历程. 该方法可用于 $\gamma$-内酰胺 13 合 成方法研究(Eq. 5). 2017 年, Xue 小组 ${ }^{[16]}$ 研究发现在催化 量的碘苯和氧化剂 $m \mathrm{CPBA}$ 原位生成三价碘介导下，芳 基酰胺类化合物 14 实现了分子内 $\mathrm{C}\left(\mathrm{sp}^{2}\right)-\mathrm{N}$ 键的偶联, 构建菲啶酮 15 骨架. 该方法底物应用范围较广，条件温 和，室温空气中即可进行，最高产率可达 $98 \%$, 且菲啶 酮类化合物是一种重要的三元环化合物，常作为医药原 料或中间体应用于制备一系列药物或活性功能分子(Eq. $6)$.

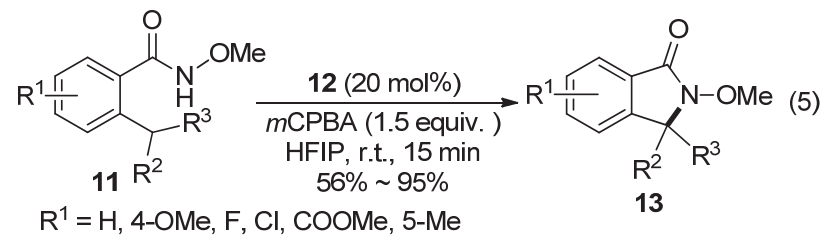

$\mathrm{R}^{1}=\mathrm{H}, 4-\mathrm{OMe}, \mathrm{F}, \mathrm{Cl}, \mathrm{COOMe}, 5-\mathrm{Me}$

$\mathrm{R}^{2}=$ cyclohexyl, Me; $\mathrm{R}^{3}=\mathrm{H}, \mathrm{Me}$

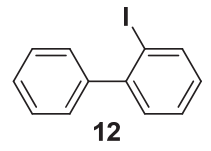

Chin. J. Org. Chem. 2020, 40, 28 39 


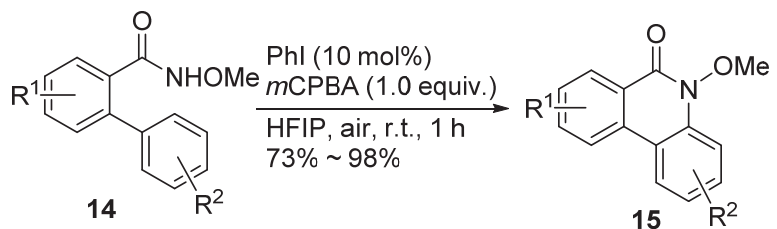

$\mathrm{R}^{1}=2-\mathrm{F}, 2-\mathrm{Ph}, 2-\mathrm{CH}_{3}, 2-\mathrm{NO}_{2}$, 2-OMe, 1-F, 1-Cl, 1-Br, 1-OMe, etc. $\mathrm{R}^{2}=3-\mathrm{Me}, 3-\mathrm{CF}_{3}, 3-\mathrm{CO}_{2} \mathrm{Me}, 3-\mathrm{F}$, etc.

2013 年, Kita 课题组 ${ }^{[17]}$ 第一次报道了原位生成三价 碘介导的分子间芳烃氧化交叉偶联反应. 该研究发现磺 酰苯胺类化合物 16 在催化剂 2,2-二碘联苯和氧化剂 $m \mathrm{CPBA}$ 作用下，芳烃类化合物经过分子间选择性的 $\mathrm{C}-\mathrm{C}$ 键偶联得到联苯类化合物 17, 产率在 54\% 99\% 之间(Eq. 7).

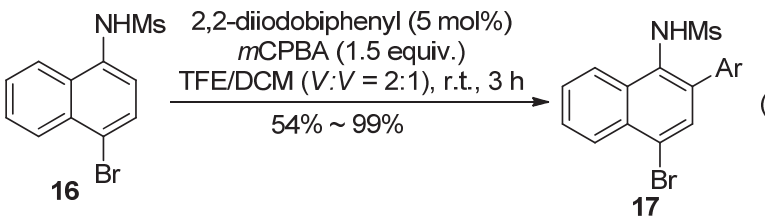

$\mathrm{ArH}=1,2,4,5-\mathrm{Me}_{4} \mathrm{C}_{6} \mathrm{H}_{2},{ }^{-}{ }^{t} \mathrm{Bu}-4-\mathrm{MeC}_{6} \mathrm{H}_{4}, 1,4-{ }^{i} \mathrm{Pr}_{2} \mathrm{C}_{6} \mathrm{H}_{4}$, toluene, naphthalene, 1-MeO-4- $\mathrm{MeC}_{6} \mathrm{H}_{4}$, etc.

$m \mathrm{CPBA}$ 作为氧化剂原位生成三价碘, 不仅可以用 于 $\mathrm{C}-\mathrm{C}, \mathrm{C}-\mathrm{O}$ 和 $\mathrm{C}-\mathrm{N}$ 键的构建, 还可以应于部分重 排反应. 早在 2010 年, Miyamoto 团队 ${ }^{[18]}$ 就报道了使用 催化量的 $\mathrm{PhI}$ 和氧化剂 $m \mathrm{CPBA}$ 原位生成高价碘介导的 霍夫曼重排反应. 反应中需要添加 $\mathrm{HBF}_{4}$ 试剂, 在反应 中起诱导作用. 碘苯、水先被氧化成四配位的三价碘 $\mathrm{PhI}^{+}\left(\mathrm{H}_{2} \mathrm{O}\right)_{2} \mathrm{OHBF}_{4}^{-}$, 然后作为氧化剂使原料酰胺完成 1 , 2 迁移, 生成 $\mathrm{RN}=\mathrm{C}=\mathrm{O}$, 再脱去 $\mathrm{CO}_{2}$ 生成伯胺, 产率较 高(Eq. 8).

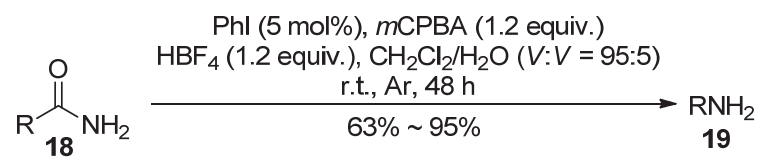

$\mathrm{R}=\mathrm{PhCH}_{3}, 4-\mathrm{MeOC}_{6} \mathrm{H}_{4} \mathrm{CH}_{2}, 4-\mathrm{MeC}_{6} \mathrm{H}_{4} \mathrm{CH}_{2}, 4-\mathrm{ClC}_{6} \mathrm{H}_{4} \mathrm{CH}_{2}$ 4- $\mathrm{CF}_{3} \mathrm{C}_{6} \mathrm{H}_{4} \mathrm{CH}_{2}, \mathrm{PhCH}_{2} \mathrm{CH}_{2}, \mathrm{C}_{6} \mathrm{H}_{13}, \mathrm{TsNH}\left(\mathrm{CH}_{2}\right)_{6}, \mathrm{NO}_{2}\left(\mathrm{CH}_{2}\right)_{5}$ $\mathrm{Br}\left(\mathrm{CH}_{2}\right)_{5}$, cyclopentyl, cyclohexyl, 1-adamantyl, etc.

2.1.2 $\mathrm{AcOOH}$ 作为氧化剂原位生成三价碘在有机合 成中的应用

过氧乙酸 $(\mathrm{AcOOH})$ 是一种过氧化物, 生活中常用于 杀菌消毒, 在化学反应中常用作烯烃的环氧化剂 ${ }^{[19]}$. 其 氧化能力低于 $m \mathrm{CPBA}$, 但在原位生成高价碘反应中仍 是常用的氧化剂, 可用于 $\mathrm{C}-\mathrm{C}, \mathrm{C}-\mathrm{O}$ 和 $\mathrm{C}-\mathrm{N}$ 键的构 建. 由于该化合物不稳定，浓度大于 $45 \%$ 或者遇高热易 爆炸, 所以 $\mathrm{AcOOH}$ 不能长时间储存, 且参与的反应加 热温度不能过高.

2011 年, Antonchick 小组 ${ }^{[20]}$ 以碘化物 21 为催化剂, 过氧乙酸为氧化剂, 使 2-氨基联苯 20 发生分子内的氧
化环合反应，构建了 $\mathrm{C}-\mathrm{N}$ 键. 该反应在室温下进行， 最高产率可达 $98 \%$ ，是合成咔唑的一种新方法(Eq. 9). 2013 年, 朱强课题组 ${ }^{[21]}$ 则报道了以碘苯、过氧乙酸构成 的催化氧化体系，通过对 $N$-苯基-2-氨基吡啶类化合物 23 进行氧化, 完成 $\mathrm{C}\left(\mathrm{sp}^{2}\right)-\mathrm{N}$ 键的构建. 该反应最高产 率可达 99\%, 可适用于多种官能团, 为苯并咪唑的基本 骨架的建立开辟了一种新的途径(Eq. 10).

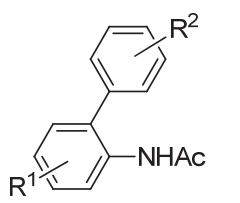

20

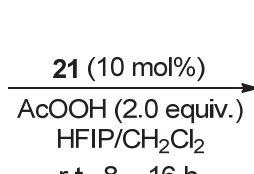

r.t., $8 \sim 16 \mathrm{~h}$ $46 \% \sim 98 \%$

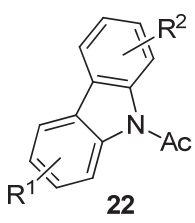

22
$\mathrm{R}^{1}=\mathrm{H}, 3-\mathrm{Cl}, 3-\mathrm{F}, 3-{ }^{t} \mathrm{Bu}, 3,4-\mathrm{F}_{2}, 3,4-\mathrm{Cl}_{2}$ $\mathrm{R}^{2}=\mathrm{H}, 3^{\prime}-\mathrm{Cl}, 4^{\prime}-\mathrm{F}, 4^{\prime}-\mathrm{Cl}, 4^{\prime}-\mathrm{I}, 4^{\prime}-\mathrm{Cl}$, etc.

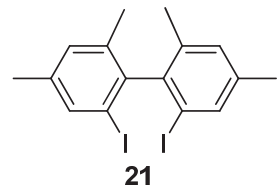

$$
\text { (10) }
$$

$\mathrm{R}^{1}=\mathrm{H}, 2-\mathrm{CH}_{3}, 2-\mathrm{F}, 2-\mathrm{NO}_{2}, 4-\mathrm{CH}_{3}, 4-\mathrm{OMe}$

$4-\mathrm{F}, 4-\mathrm{Cl}, 4-\mathrm{Br}, 4-\mathrm{I}, 4-\mathrm{NO}_{2}, 4-{ }^{t} \mathrm{Bu}$

$\mathrm{R}^{2}=\mathrm{H}, 3-\mathrm{F}, 3-\mathrm{Cl}, 3-\mathrm{CH}_{3}, 4-\mathrm{CH}_{3}, 4-\mathrm{CF}_{3}, 6-\mathrm{CH}_{3}$

在 2014 年, Martin 课题组 ${ }^{[22]}$ 报道了在非金属条件 下, 取代碘苯作为催化剂, 过氧乙酸为氧化剂原位生成 三价碘介导的 $\mathrm{sp}^{2}$ 或 $\mathrm{sp}^{3} \mathrm{C}-\mathrm{H}$ 键和羧酸之间的偶联反应. 该方法成功构建了 $\mathrm{C}-\mathrm{O}$ 键，实现了分子内酯化反应， 且条件温和，底物使用范围广泛(Eqs. 11, 12).
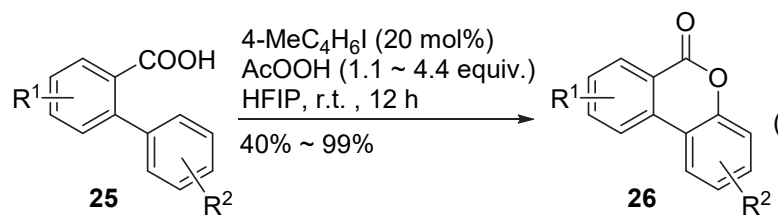

$\mathrm{R}^{1}=\mathrm{H}, 5-\mathrm{NO}_{2}, 4-\mathrm{CF}_{3}, 4-\mathrm{AcO}, 3-\mathrm{Me}, 4,5-(\mathrm{MeO})_{2}$, etc. $\mathrm{R}^{2}=\mathrm{H}, 4-\mathrm{Me}, 2-\mathrm{CO}_{2} \mathrm{Me}, 4-\mathrm{CO}_{2} \mathrm{Me}, 4-\mathrm{F}, 3,4-(\mathrm{MeO})_{2}$, etc.

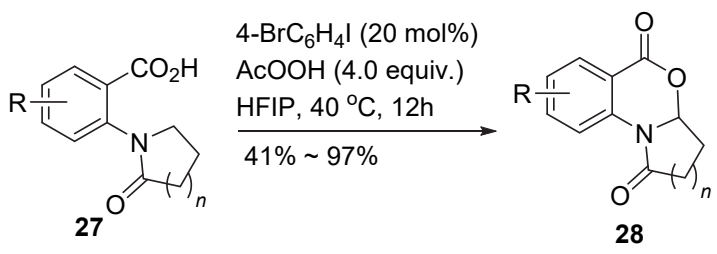

$\mathrm{R}=\mathrm{H}, 4-\mathrm{Cl}, 4-\mathrm{Me}, 4-\mathrm{CO}_{2} \mathrm{H}, 5-\mathrm{F}, 5-\mathrm{Cl}, 5-\mathrm{Br}, 5-\mathrm{I} ; n=1 \sim 2$

原位生成三价碘介导的 $\mathrm{C}-\mathrm{O}$ 键偶联反应同样可以 用于化合物的磺化反应. 2017 年, 沈超课题组 ${ }^{[23]}$ 报道了 在碘苯-过氧乙酸组合作用下喹啉酰胺类化合物 $\mathbf{2 9}$ 的磺 
化反应. 该反应在室温下溶剂 HFIP 中进行. 经过氧化 之后，以 $72 \% \sim 95 \%$ 的收率得到一系列芳基磺酸酯类化 合物 31. 合成得到的部分磺化喹诺酮类化合物可用于 生物和医药领域(Eq. 13).

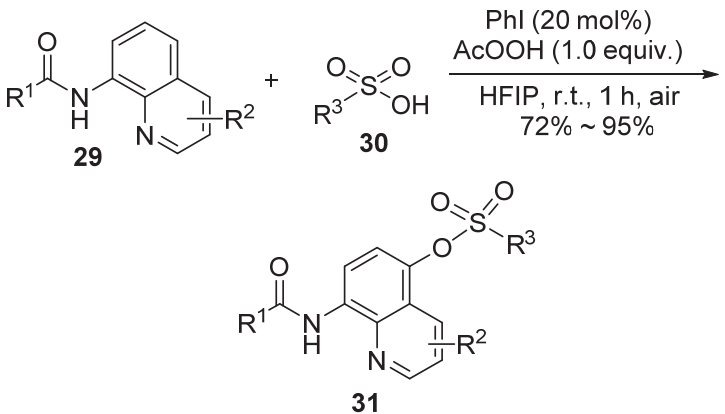

$\mathrm{R}^{1}=\mathrm{Ph}, 3-\mathrm{MeC}_{6} \mathrm{H}_{4}, 2,4-\mathrm{Cl}_{2} \mathrm{C}_{6} \mathrm{H}_{3}$, cyclohexyl, pyridyl, etc $\mathrm{R}^{2}=\mathrm{H}, 2-\mathrm{Me}$

$\mathrm{R}^{3}=\mathrm{C}_{6} \mathrm{H}_{5}, \mathrm{Me}, \mathrm{CF}_{3}$, naphthyl

2.1.3 NBS 作为氧化剂原位生成三价碘在有机合成 中的应用

$N$-溴代琥珀酰亚胺(NBS)一般用于有机化学中的自 由基取代、亲电加成、溴代反应以及氧化反应. 在原位生 成高价碘反应中, NBS 不仅可以作为氧化剂还可以作为 溴的引入剂, 而且与其它过氧化物相比, 无爆炸危险, 更加安全. 目前文献报道 NBS 参与的原位生成高价碘 反应多为氧化反应、重排反应和溴代反应. 在 2006 年, Braddock 课题组 ${ }^{[24]}$ 报道了以邻位取代的碘苯作为有机 催化剂, NBS 作为氧化剂, 实现了烯烃基羧酸的溴代内 酯化反应. 随后 Gulder 课题组 ${ }^{[25 ~ 28]}$ 报道了一系列 NBS 作为氧化剂原位生成高价碘介导的一系列反应.

2012 年, Gulder 课题组 ${ }^{[25]}$ 研究发现, 甲基丙烯胺化 合物 32 在 NBS 和催化剂邻碘苯酰胺衍生物 33 的共同 作用下, 完成了溴代环合反应, 得到目标产物 3,3-二取 代的吲哚类化合物 34, 产率在 59\% 94\%之间. 该环合 方法已经用于乙酰胆碱酯酶抑制剂毒扁豆碱的制备中 (Eq. 14).

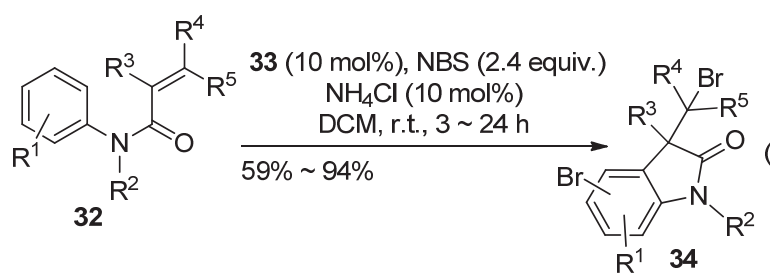

$\mathrm{R}^{1}=4-\mathrm{MeO}, 4-\mathrm{Me},{ }^{\mathrm{t}}{ }^{\mathrm{t}} \mathrm{Bu}, 2-\mathrm{Me}, 4-\mathrm{TBSO}, \mathrm{CH}_{3} \mathrm{CO}$, etc. $\mathrm{R}^{2}=\mathrm{Me}, \mathrm{Bn} ; \mathrm{R}^{3}=\mathrm{Me}, \mathrm{CH}_{2} \mathrm{CH}_{2} \mathrm{OTBS} ; \mathrm{R}^{4}, \mathrm{R}^{5}=\mathrm{H}, \mathrm{Me}$<smiles>O=C(O)CNC(=O)c1ccccc1I</smiles>

$\mathrm{NBS}$ 氧化剂不仅可以用于 $\mathrm{C}-\mathrm{C}$ 键氧化偶联反应,
而且还可以用于重排反应中. 2015 年, Gulder 课题组 ${ }^{[26]}$ 报道了原位生成三价碘介导的酰亚胺类化合物 35 发生 重排反应，得到了一系列的 $\alpha, \alpha$-二取代- $\alpha$-差基羧酰胺类 化合物 36 (Eq. 15). 反应在室温下进行, 产率可高达 98\%，并且在未用手性添加剂情况下，诱导产生单一的 手性化合物. 值得一提的是, 该报道用 ${ }^{1} \mathrm{H} \mathrm{NMR},{ }^{13} \mathrm{C}$ NMR 和 ESI-MS 捕捉到了三价碘 $\mathbf{3 7}$, 证实了原位生成的 三价碘化合物参与了催化过程, 其反应机理如 Scheme 2 所示. 这为将来根据不同反应的特点设计和选择有效的 催化剂提供了理论支撑.

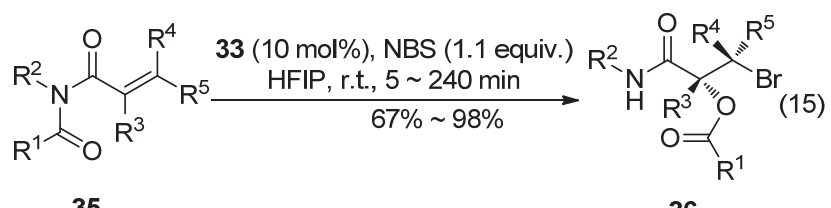

35

36

$\mathrm{R}^{1}=\mathrm{Ph},{ }^{t} \mathrm{Bu} ; \mathrm{R}^{2}=\mathrm{Me}, \mathrm{Ph}, 1$-naphthyl, $2-\mathrm{MeOC}_{6} \mathrm{H}_{4}, \mathrm{Bn}, \mathrm{PhCHMe},{ }^{i} \mathrm{Pr}$ $\mathrm{R}^{3}=\mathrm{Me}, \mathrm{Ph}, \mathrm{Bn} ; \mathrm{R}^{4}=\mathrm{H}, \mathrm{Me} ; \mathrm{R}^{5}=\mathrm{H}, \mathrm{Me}$

基于前面的报道, 2016 年, 该课题组 ${ }^{[27]}$ 用 Eq. 14 中 相同的原料、氧化剂和催化剂, 对一锅法制备异丝氨酸 类衍生物方法进行了深入的研究. 反应中原料酰胺类化 合物 45 在氧化剂 NBS 和催化剂取代碘苯 33 作用下发 生溴化重排反应，得到 $\beta$-卤代羧基酰胺中间体 46(即 Scheme 2 中产物), 而后加入碱 $\mathrm{CsF}$, 溶剂加热回流得到 $\beta$-内酰胺类环合中间体 47 , 最后在酸性 TFA 作用下开环 得到一系列异丝氨酸衍生物 48. 该方法利用原位生成 高价碘策略，成功地实现了一锅四步的反应，为 $\beta$-内酰 胺类化合物及异丝氨酸类化合物的合成提供了新途径 (Scheme 3).

Gulder 课题组 ${ }^{[28]}$ 对原位生成三价碘介导的区域选 择性的双卤代反应也进行了研究. 反应选用 $N$-丁基-邻 苯酰亚胺 50 作为催化剂, NBS 或者 KBr-Oxone 组合作 为氧化剂来代替溴单质, 将一系列烯烃或炔烃 49 转化 为反式的双溴代化合物 51, 发现产物中未发生芳香溴 化. 进一步研究表明，在相同的反应条件下，当反应中 的氧化剂为 KCl-Oxone 时, 可以实现炔烃或者烯烃的双 氯代, 产率中等偏上(Eq. 16).

2.1 .4 其它氧化剂原位生成三价碘在有机合成中的 应用

可用于原位生成高价碘的氧化剂有很多种，除常用 的 $m \mathrm{CPBA}, \mathrm{AcOOH}$ 和 $\mathrm{NBS}$ 外，另有文献报道了 $\mathrm{NaBO}_{3}$ • $\mathrm{H}_{2} \mathrm{O}$ 、Selectfluor (1-氯甲基-4-氟-1,4-重氮化二环[2.2.2] 辛烷双(四氟硣酸)盐)以及 Oxone(过硫酸氢钾复合盐) 等 都可以作为氧化剂参与反应, 分别完成 $\mathrm{C}-\mathrm{C}$ 和 $\mathrm{C}-\mathrm{O}$ 键的氧化偶联. 而且 Oxone 参与原位生成高价碘可以使 酰胺类化合物完成霍夫曼重排反应，且产率较高. 


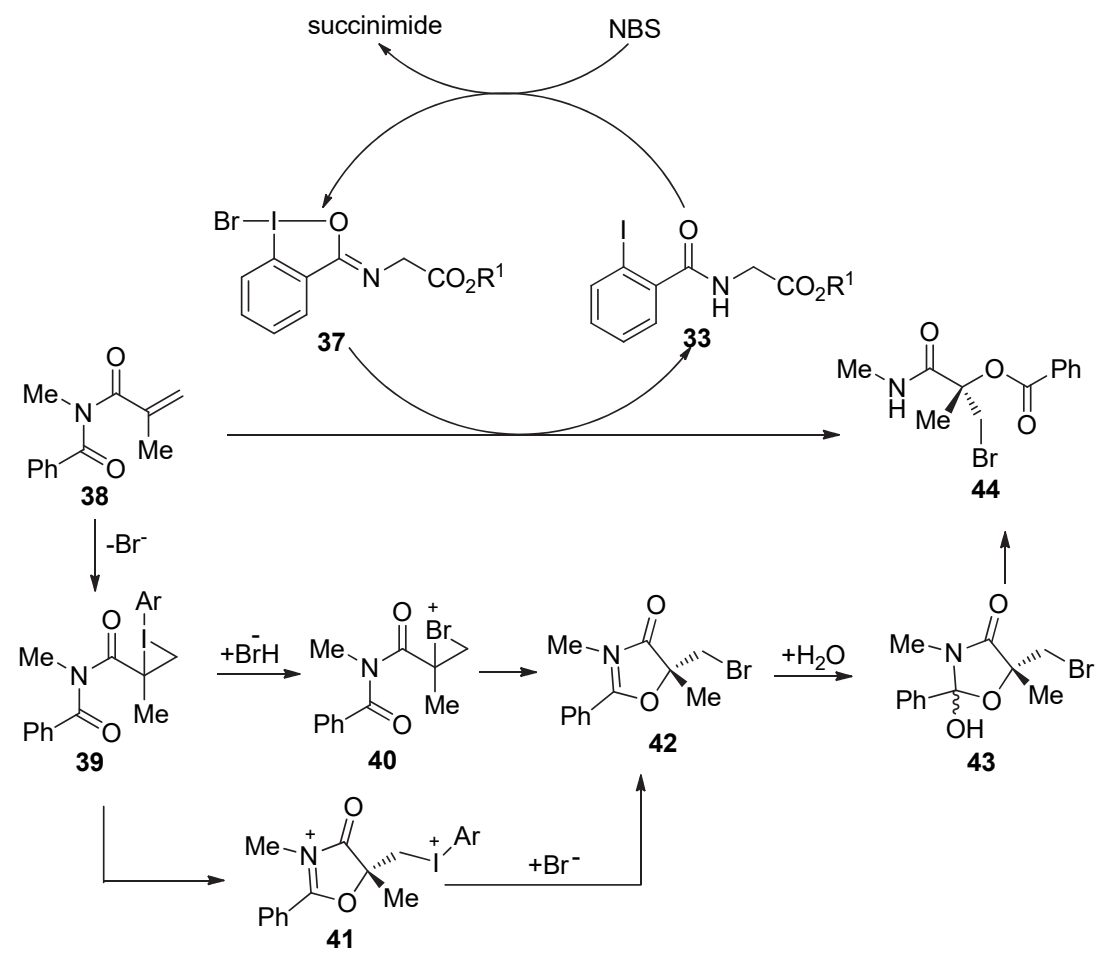

图式 2 NBS 作为氧化剂原位生成三价碘介导的重排反应

Scheme 2 NBS as an oxidant in situ to generate iodine(III) reagents-mediated rearrangement reaction

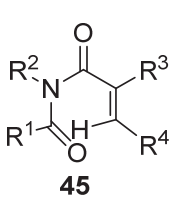

33 (10 mol\%), NBS (1.1 equiv.) CsF (7.0 equiv.) $30 \mathrm{~mol} \% \mathrm{TEBACl}$, aq. $\mathrm{NH}_{4} \mathrm{Cl}$

THF/DCM $(V: V=1: 1)$, reflux TFA, THF/DCM $(V: V=1: 1)$, reflux $48 \% \sim 71 \%$

bromination/ rearrangement

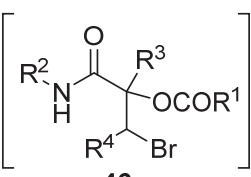

46

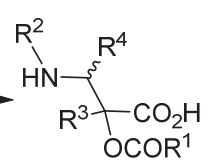

48

ring-opening

$\mathrm{R}^{1}=\mathrm{Ph}$, neopentyl; $\mathrm{R}^{2}=\mathrm{Me}, \mathrm{Ph}, \mathrm{Bn},{ }^{i} \mathrm{Pr}$, etc. $\mathrm{R}^{3}=\mathrm{Me}, \mathrm{Ph}, \mathrm{Bn}, \mathrm{Et}, n$-Hex, etc.; $\mathrm{R}^{4}=\mathrm{H}, \mathrm{Me}$

图式 $3 \mathrm{NBS}$ 作为氧化剂原位生成三价碘介导的异丝氨酸类 衍生物的合成

Scheme 3 NBS as an oxidant in situ to generate iodine(III) reagents-mediated synthesis of isoflavin derivatives

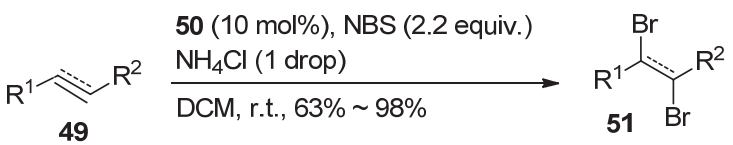

$\mathrm{R}^{1}={ }^{n} \mathrm{C}_{6} \mathrm{H}_{13},{ }^{n} \mathrm{C}_{4} \mathrm{H}_{9}, \mathrm{Et}, \mathrm{Ph}, \mathrm{CO}_{2} \mathrm{Me}$, etc.

$\mathrm{R}^{2}=\mathrm{H}, \mathrm{CH}_{2} \mathrm{OH}, \mathrm{NO}_{2}, \mathrm{CO}_{2} \mathrm{Me}, \mathrm{COMe}, \mathrm{Me}$, Et, etc.<smiles>CCCCNC(=O)c1ccccc1I</smiles>

\section{2 无机磑盐或者单质磑作为催化剂原位生成高价磑 在合成中的应用}

一些无机碘盐或者碘单质同样可以代替芳基碘作 为催化剂在反应中生成高价碘 ${ }^{[10]}$, 相关文献中的机理 推测以生成三价碘中间体最为常见. 该类反应中常用的 碘盐有四丁基碘化铵(TBAI)、 KI 和 $\mathrm{NaI}$, 这些碘盐在反 应中通常被氧化为一价的 $\mathrm{IO}^{-}$或者三价的 $\mathrm{IO}_{2}^{-}$, 后者随 后参与氧化偶联反应. 而碘单质则是在反应中先形成自 由基, 再被氧化为高价碘参与反应(表 1).

2011 年, Ishihara 课题组 ${ }^{[33]}$ 报道了分子内和分子间 的羰基 $\alpha$-位氧酰化反应. 使用 TBAI 作为催化剂, 过氧 化氢作为氧化剂完成分子间和分子内酯化反应. 酤类、 醛类、二羰基化合物都可以发生反应，且反应条件温和， 产率较高，最高可达 99\% (Eqs. 17, 18).

2011 年, 于炜和韩丙课题组 ${ }^{[34]}$ 报道了一种原位生 成高价碘介导的 $\mathrm{C}-\mathrm{N}$ 偶联反应. 原料氨基吡啶 56 与 $\beta$ 酮酯或 1,3-二酮 57 在催化量的 TBAI 和过氧叔丁醇 (TBHP)作用下反应, 生成了一系列的咪唑并 $[1,2-a$ 吡啶 58 (Eq. 19).

同年，魏运洋课题组 ${ }^{[35]}$ 将四丁基碘化铵和过氧化 氢在反应体系中原位生成高价碘, 以邻苯二胺 59 和苯 甲醛 60 为原料构建苯并咪唑 61 . 值得一提的是, 反应 过程首次用 ESI-MS 检测到了中间体三价碘 $\left[\mathrm{Bu}_{4} \mathrm{~N}\right]^{+}$$\left[\mathrm{IO}_{2}\right]^{-}$, 这为反应机理提供了可信的实验证据. 该反应 
表 $1 \quad \mathrm{NaBO}_{3} \cdot \mathrm{H}_{2} \mathrm{O}$, selectfluor, oxone 作为氧化剂原位生成三价碘在有机合成中的应用

Table 1 Application of $\mathrm{NaBO}_{3} \cdot \mathrm{H}_{2} \mathrm{O}$, selectfluor, oxone as oxidant in situ to generate trivalent iodine in organic synthesis

序号

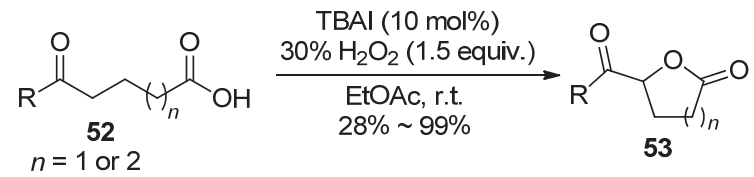

$\mathrm{R}=\mathrm{Ph}, 1-\mathrm{Naphthyl}, 2-\mathrm{FC}_{6} \mathrm{H}_{4}, 2-\mathrm{MeOC}_{6} \mathrm{H}_{4}, 2-\mathrm{Py}, \mathrm{Me}$, etc

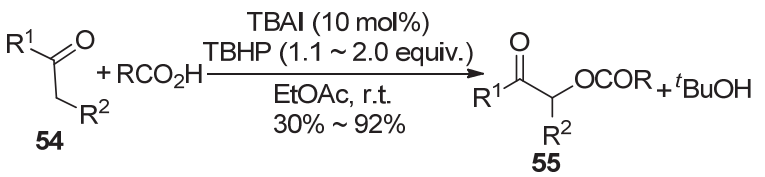

$\mathrm{R}^{1}=\mathrm{Me}, \mathrm{Ph}, 4-\mathrm{FC}_{6} \mathrm{H}_{4}, 3,4-\mathrm{Cl}_{2} \mathrm{C}_{6} \mathrm{H}_{3}, 4-\mathrm{MeC}_{6} \mathrm{H}_{4} ; \mathrm{R}^{2}=\mathrm{Me}, \mathrm{Pr}$, etc.

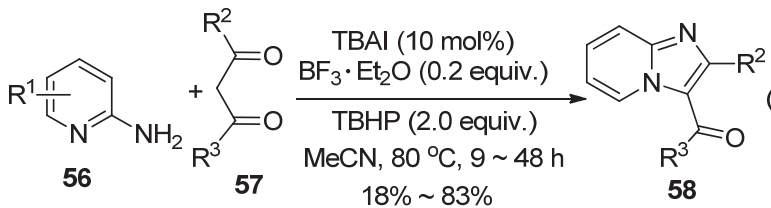

$\mathrm{R}^{1}=\mathrm{H}, 5-\mathrm{Me}, 4-\mathrm{Me}$, 3-Me, 5-Cl, 4-Cl; $\mathrm{R}^{2}=\mathrm{Ph}, 4-\mathrm{EtC}_{6} \mathrm{H}_{4}$, 3- $\mathrm{MeOC}_{6} \mathrm{H}_{4}, 4-\mathrm{ClC}_{6} \mathrm{H}_{4},{ }^{n} \mathrm{Pr}, \mathrm{Me}$

$\mathrm{R}^{3}=\mathrm{Et}, \mathrm{Me}$

不使用酸和过渡金属, 且唯一的副产物是水(Eq. 20).<smiles>[R]c1ccc(-c2nc3ccc([R])cc3[nH]2)cc1</smiles>

$\mathrm{R}^{1}=4-\mathrm{Me}, 4-\mathrm{NO}_{2}$, etc.; $\mathrm{R}^{2}=4-\mathrm{Cl}, 4-\mathrm{NO}_{2}, 4-\mathrm{OMe}, 4-\mathrm{OH}$, etc.

Togo 课题组 ${ }^{[12]}$ 已经报道了过碘苯作为催化剂介导 的羰基 $\alpha$ 位苯甲磺酰化. 在此基础上, 2014 年, Shafir 课 题组 ${ }^{[36]}$ 提出 $\mathrm{KI}$ 或者 $\mathrm{I}_{2}$ 可以代替碘苯完成此反应, 且产
率中等(Eq. 21). 随后, 2015 年张敏和苏伟平课题组 ${ }^{[37]}$ 报 道了 NaI- $m$ CPBA 组合原位生成高价碘介导的酮类化合 物 62 的 $\alpha$-烷氧基化反应. 该反应最关键的步骤是由 $\mathrm{NaI}$ 和 $m \mathrm{CPBA}$ 催化氧化酮生成中间体 $\alpha$-碘酮，随后该中间 体上的碘被甲醇亲核取代得到最终产物 65 (Eq. 22).

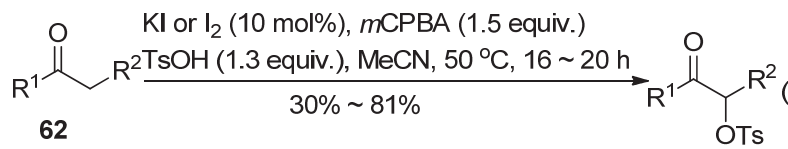

$\mathrm{R}^{1}=\mathrm{Ph}, 4-\mathrm{MeC}_{6} \mathrm{H}_{4}, 4-\mathrm{BrC}_{6} \mathrm{H}_{4}, 4-\mathrm{ClC}_{6} \mathrm{H}_{4}, 4-\mathrm{NO}_{2} \mathrm{C}_{6} \mathrm{H}_{4}$, Et 63 $\mathrm{R}^{2}=\mathrm{H}, \mathrm{Me}$

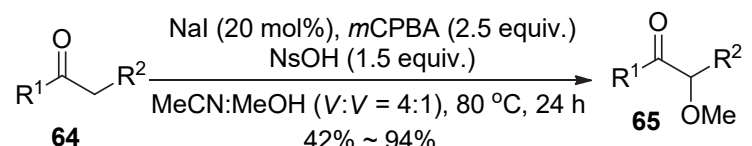

$\mathrm{R}^{1}=\mathrm{Ph}, 4-\mathrm{MeC}_{6} \mathrm{H}_{4}, 3-\mathrm{MeC}_{6} \mathrm{H}_{4}, 4-\mathrm{BrC}_{6} \mathrm{H}_{4}, 4-\mathrm{ClC}_{6} \mathrm{H}_{4}, 3-\mathrm{ClC}_{6} \mathrm{H}_{4}$ 4- $\mathrm{FC}_{6} \mathrm{H}_{4} 3-\mathrm{FC}_{6} \mathrm{H}_{4}$, 2-thienyl, etc.

$\mathrm{R}^{2}=\mathrm{Me}, \mathrm{Et}, \mathrm{Ph}$

2018 年, Reiher 和 Muniz 等 ${ }^{[38]}$ 的研究表明，酰胺类 脂肪烃化合物 66 可在原位生成高价碘介导下发生分子 内的碳一氮键氧化偶联，形成四氢吡咯类化合物 67. 该 反应以单质碘作为催化剂, 完成了 Hofmann-Löffler 反 应(Eq. 23).

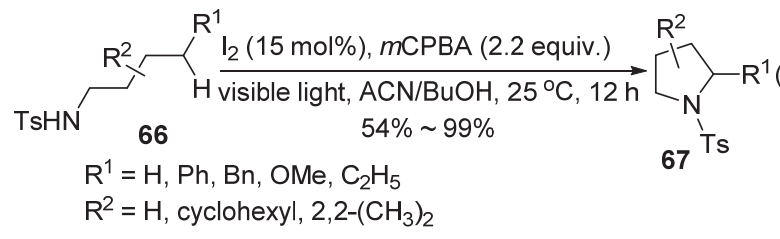




\section{3 原位生成五价碘试剂在合成中的应用}

原位生成五价碘试剂与原位生成三价碘过程类似, 即催化量的碘试剂在氧化剂作用下, 被氧化成五价碘, 后者参与氧化反应. 两者的区别主要在于使用的催化剂 不同. 原位生成五价碘大部分使用邻碘苯甲酸及其衍生 物为催化剂, 被氧化后生成五价碘中间体 IBX (2-碘酰 基苯甲酸)或者 $\mathrm{PhIO}_{2}$, 后者参与氧化反应(机理如 Scheme 4, 以醇氧化为羰基化合物为例). 因为五价碘在 大部分溶剂中溶解度不高, 目前原位生成五价碘试剂应 用范围较窄，大部分用于醇类氧化反应.

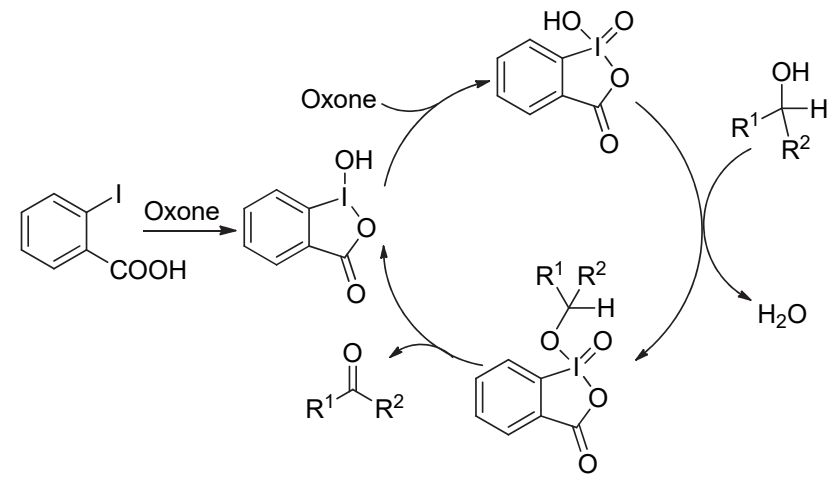

图式 4 原位生成五价碘化合物的策略示意图

Scheme 4 In-situ formation of hypervalent iodine(V) reagent

2005 年, Vinod 课题组 ${ }^{[39]}$ 首次实现了原位生成五价 碘介导的醇类的氧化. 该反应中催化量的 2-碘苯甲酸和 氧化剂 Oxone 加热到 $70{ }^{\circ} \mathrm{C}$ 生成中间体五价碘 $\mathrm{IBX}$, 随 后在 IBX 的氧化作用下, 伯醇 68 转化为对应的酸 69 , 最高产率可达 97\% (Eq. 24). 2006 年, Giannis 课题组 ${ }^{[40]}$ 报道了类似的反应. 反应中使用混合溶剂 $\mathrm{MeCN} / \mathrm{H}_{2} \mathrm{O}$ $(V: V=2: 1)$, 因此添加了催化量的 $\mathrm{Bu}_{4} \mathrm{NHSO}_{4}$ 作为相 转移催化剂, 使一系列茮醇 70 以 $72 \%$ ～93\%的产率转化 为苯甲醛 71. 同时, 该反应仲醇只能转化为酮类, 不可 进一步转化为酸(Eq. 25).
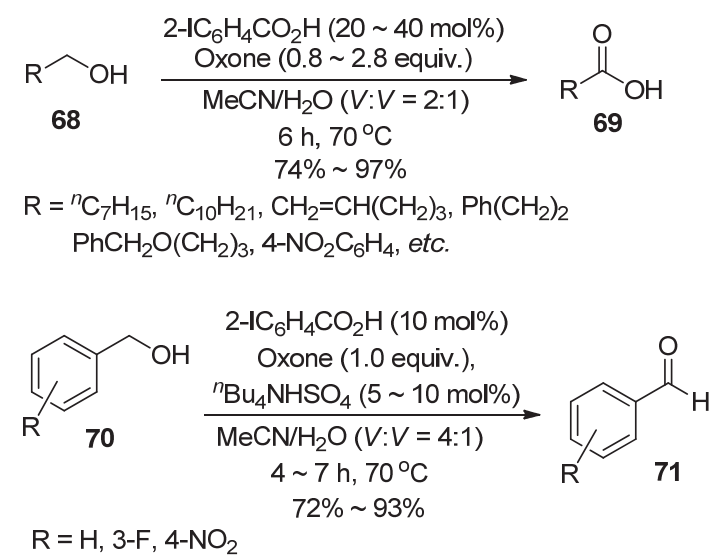

2009 年, Ishihara 课题组 ${ }^{[41]}$ 研究发现, 醇类可以在 2-
碘苯磺酸(IBS)与 Oxone 原位生成五价碘的介导下氧化 成酮及酸. 此外, 环己醇 72 在相似条件下，使用过量 Oxone 可氧化成相应的环烯酮 73, 产率中等(Eq. 26). 同 年, 该课题组 ${ }^{[42]}$ 又报道了 IBS 和 Oxone 催化介导下, 叔 丁基醇 74 氧化重排成烯酤 75 (Eq. 27).

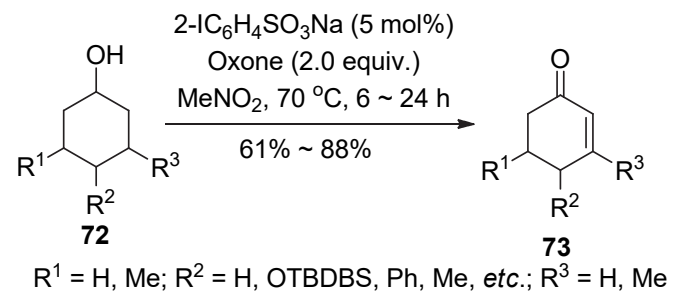

$$
\begin{aligned}
& \mathrm{R}^{1}={ }^{n} \mathrm{Bu}, \mathrm{Ph} ; \mathrm{R}^{2}=\mathrm{Me}, \mathrm{Ph} ; \mathrm{R}^{3}=\mathrm{Me},{ }^{\mathrm{I} P r}, \mathrm{Ph} \\
& \begin{array}{c}
\mathrm{K}_{2} \mathrm{CO}_{3}(0.5 \text { equiv. }), \mathrm{EtOAc}, 60{ }^{\circ} \mathrm{C} \\
\text { Oxone (1.0 equiv. })
\end{array}
\end{aligned}
$$

2009 年, Yusubov 课题组 ${ }^{[43]}$ 报道了以催化量的 PhI, $\mathrm{RuCl}_{3}$ 以及氧化剂 Oxone 原位生成五价碘 $\mathrm{PhIO}_{2}$ 的方法. 反应体系中, $\mathrm{PhI}$ 和 Oxone 首先生成 $\mathrm{PhIO}$, 随后 $\mathrm{PhIO}$ 在 $\mathrm{RuCl}_{3}$ 作用下发生歧化反应产生 $\mathrm{PhIO}_{2}$. 反应中各类醇 可以被氧化为相应的酮或羧酸，产率较高 $(80 \%$ 以上). 值得注意的是，该反应中丙基苯以及部分芳环相邻 $\alpha$ 位 的 $\mathrm{C}-\mathrm{H}$ 键同样可以被氧化为羰基(Eq. 28).

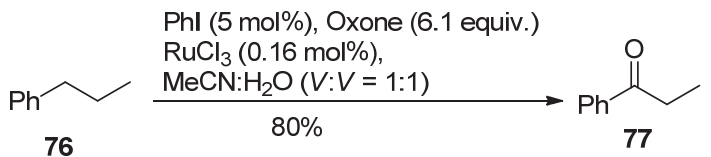

\section{4 原位生成手性高价碘试剂在合成中的应用}

众所周知，手性化合物在医药、农药、激素和食品添 加剂等精细化工以及具有生理活性物质的生产上具有 广泛的应用，但是手性化合物在合成过程中会面临手性 拆分成本高，工业化难的问题. 因此研究有机高价碘试 剂介导的不对称合成反应具有重要的实用价值. 该类方 法即通过外加氧化剂将带有手性的芳基碘(碘为负一价) 氧化成手性高价碘化物，进而参与催化不对称氧化反 应. 该方法可以合成单一构型的手性化合物，且无需外 加的手性配体协同参与手性控制, 转化途径绿色环保, 但是氧化过程的效率和立体选择性还有待提高.

\section{1 原位生成手性高价碘试剂在 $\mathrm{C}\left(\mathrm{sp}^{3}\right)-\mathrm{H}$ 官能团化 反应中的应用}

$\mathrm{C}\left(\mathrm{sp}^{3}\right)-\mathrm{H}$ 键官能团化是一种非常重要的有机反应 类型，原位生成的手性高价碘可以介导苄基、羰基的 $\alpha$ 
位等活性亚甲基的氧化官能团化反应，并且诱导产生单 一手性化合物.

2007 年, Wirth 课题组 ${ }^{[44]}$ 报道了原位生成手性高价 碘介导的对映选择性的合成反应. 该反应以 0.1 equiv. 的 取代手性碘苯 79 作为催化剂, 3 equiv. $m$-CPBA 作为氧 化剂, 在室温条件下实现了酮类化合物 78 的 $\alpha$ 位不对称 甲磺酰基化反应. 该反应产率适中, 缺点是立体选择性 不高( $24 \% \sim 28 \%)$, 但是该方法为 $\mathrm{C}\left(\mathrm{sp}^{3}\right)-\mathrm{H}$ 官能团化的 不对称催化反应提供了研究基础(Eq. 29). 随后熊燕课 题组 ${ }^{[45]}$ 报道了在原料 $\alpha$-取代- $\beta$-酮酸 81 的 C-2 位置引入 羟基或者苯甲磺酸的反应, 实现了 $\mathrm{C} 2$-位立体中心的转 化, 最高 $e e$ 值达到 $69 \%$, 但是该类反应仍需提高对应异 构体的产率(Eq. 30).
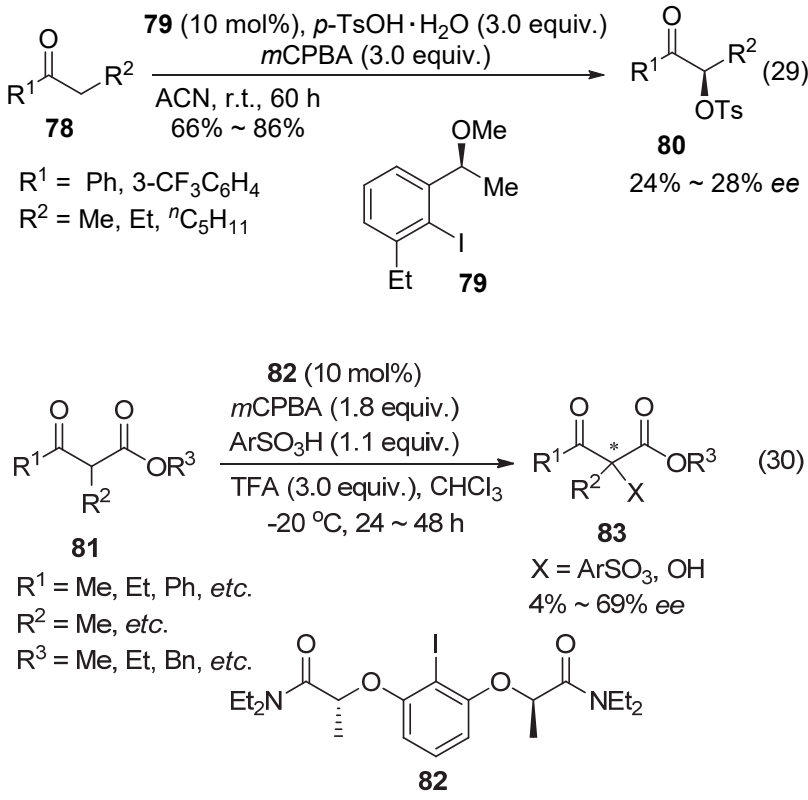

2018 年, Rueping 课题组 ${ }^{[46]}$ 也报道了类似的反应. 狮满酮 $\beta$-酮酯类化合物 84 可以在原位生成的高价碘体 系中实现羰基 $\alpha$ 位的氟化, 得到手性产物 86 (Eq. 31), ee 值高达 $91.5 \%$.
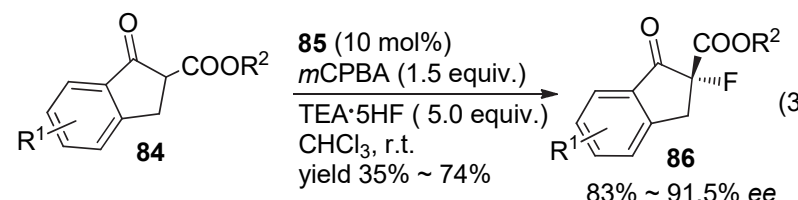

$\mathrm{R}=2-\mathrm{Br} ; 3-\mathrm{Cl}, \mathrm{Br}, \mathrm{F}, \mathrm{CF}_{3}, \mathrm{Me} ; 4-\mathrm{Cl}, \mathrm{Br}$, OMe. etc

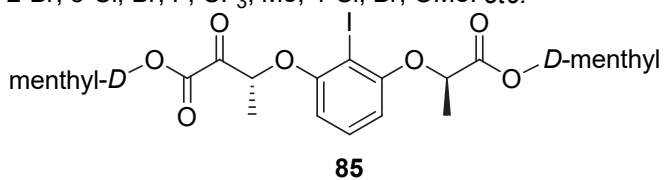

\section{2 原位生成手性高价碘介导的去芳构化反应}

去芳构化为苯酚或苯胺的芳环失去芳香性的过程, 去芳构化的过程中旧键断去, 新键生成, 易于构建螺
环、桥环或者稠环等复杂环系. 实现芳构化的方法有多 种，如氧化、加成、取代、还原等反应，其中原位生成 高价碘试剂介导的氧化芳构化是比较经典的，该过程主 要经历了原位生成高价碘、高价碘试剂与酚羟基的络 合、亲核试剂进攻芳环去芳构化反应等过程。

据 Harned 课题组 ${ }^{[47]}$ 研究表明，手性碘代芳烃是可 以用作分子间氧化偶联反应的催化剂，该反应可以使酚 类化合物 87 选择性氧化脱芳构化并引入羟基，得到相 应的环己二烯酮类衍生物 $\mathbf{8 9}$, 产率和对映选择性中等. 该反应中的催化剂也可用于酚类化合物分子内的螺环 化反应(Eq. 32)

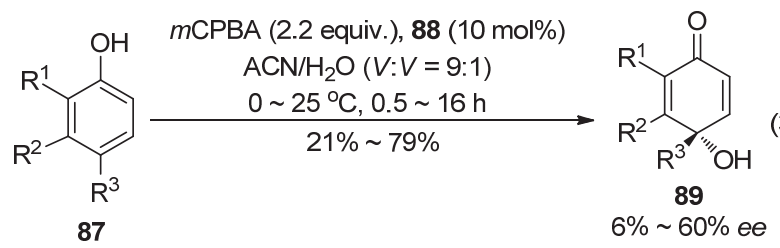

$\mathrm{R}^{1}=\mathrm{H}, \mathrm{Me}, \mathrm{Cl}, \mathrm{Br}, \mathrm{TMS}, \mathrm{TBS}$, TIPS; $\mathrm{R}^{2}=\mathrm{H}, \mathrm{Me}, \mathrm{OMe} ; \mathrm{R}^{3}=\mathrm{Me},{ }^{\mathrm{i}} \mathrm{Pr}$<smiles>CNC(=O)C1OC2(CC[B]c3cccc(I)c32)O[C@@H]1C(=O)NC</smiles>

原位生成手性高价碘的使用能够实现酚类化合物 的芳构化，诱导产生螺环中心对映选择性，这类反应已 经被很多课题组报道 ${ }^{[47 ~ 52]} .2007$ 年, Kita 课题组 ${ }^{[48]}$ 选择 具有螺二狮骨架的手性三价碘作为氧化剂, 实现了原料 荎酚类化合物的脱芳构化. 随后，该组 ${ }^{[49]}$ 改进了手性催 化方法. 使用邻位取代的螺二狮芳基碘苯 92 作为催化 剂, 在 $m \mathrm{CPBA}$ 的氧化作用下原位生成中间体三价碘 $\mathbf{9 1}$, 后者将原料萗酚 90 氧化为螺环化合物 93, 该反应在 $0{ }^{\circ} \mathrm{C}$ 下进行，对映选择性良好，ee 值可达 92\% (Eq. 33). 在 Kita 课题组研究基础上, 2010 年 Ishihara 课题组 ${ }^{[50]}$ 研

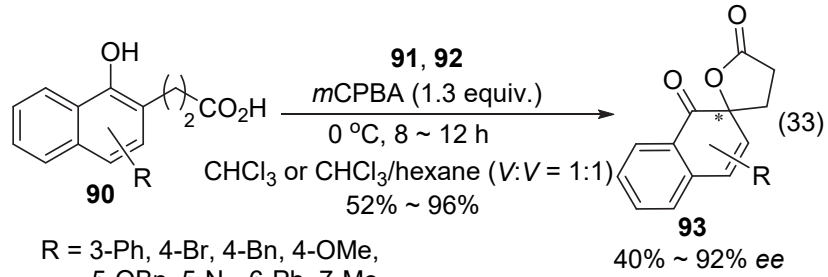
5-OBn, 5- $\mathrm{N}_{3}$, 6-Ph, 7-Me

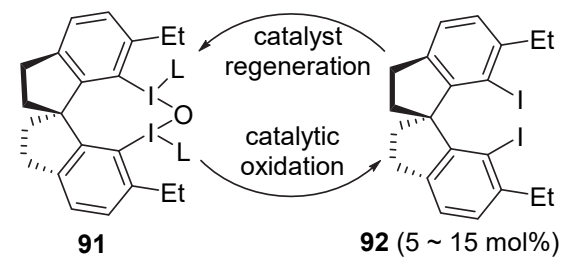


究表明, 使用一种 $C 2$ 轴对称的手性芳基碘化合物 95 作 为催化剂, 同样可以实现原料的去芳构化, 并且 $e e$ 值很 高, 在 $83 \% \sim 90 \%$ 之间(Eq. 34). 最近, Kita 课题组 ${ }^{[51]}$ 研 究发现 2,2-二甲基 8,8-二碘荟衍生物 97 可以作为催化 剂, 原位生成手性高价碘, 并完成了菜酚 96 的螺环化反 应，且对映选择性良好(Eq. 35)。

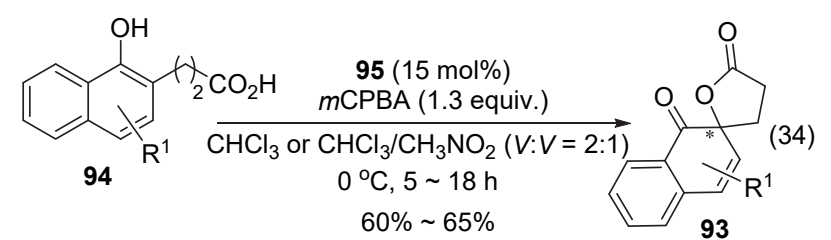

$\mathrm{R}^{1}=4-\mathrm{Me}, 4-\mathrm{Cl}, 4-\mathrm{Ph}, 4-\mathrm{COPh}$,

$83 \% \sim 90 \%$ ee

4-OMe, 6-OMe, 3-OMe, etc.

$\mathrm{R}^{2}=$ CONHMes

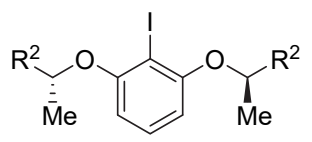

95

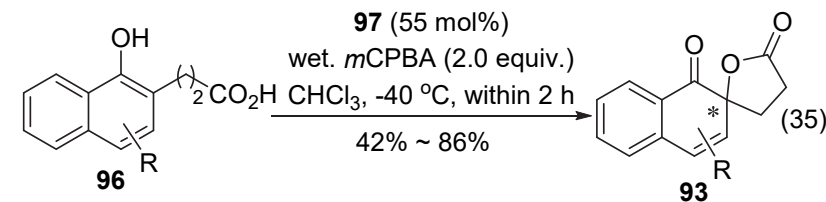

$\mathrm{R}=3-\mathrm{OH}, 4-\mathrm{Cl}, 4-\mathrm{Et}, 4-\mathrm{OMe}, 5-\mathrm{Br}, 5-\mathrm{N}_{3}, 5-\mathrm{OBn}, 6-\mathrm{N}_{3} \quad \stackrel{93}{59 \%} \sim 76 \%$ ee<smiles>[O-]c1cccc(I)c1-c1c(I)cccc1I</smiles>

2015 年, 龚流柱课题组 ${ }^{[52]}$ 报道了另外一种合成手 性螺环化合物的方法. 以 Ishihara 课题组使用的手性芳 基碘化物 95 作为催化剂, 在反应体系中原位生成手性 高价碘, 将原料-羟基- $N$-芳基-萗甲酰胺衍生物 98 进行 去芳构化, 构建 $\mathrm{C}-\mathrm{C}$ 键, 得到螺杂环产物 99, 该方法 产率高, 对映性好, 但是要在低温下 $-30{ }^{\circ} \mathrm{C}$ 下进行反 应(Eq. 36).

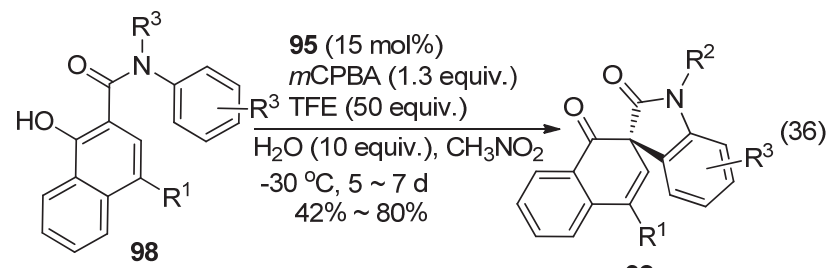

99
$80 \%$

$\mathrm{R}^{1}=\mathrm{H}, \mathrm{Br} ; \mathrm{R}^{2}=\mathrm{Me}, \mathrm{Et}, \mathrm{Ph} ; \mathrm{R}^{3}=\mathrm{H}, 3,5-\mathrm{Me}_{2}, 4-\mathrm{Me}, 4-\mathrm{OMe}$, etc.

\section{3 原位生成手性高价碘介导的氧化环合反应}

2014 年, Wirth 课题组 ${ }^{[53]}$ 以手性碘苯 101 作为催化
剂，将原位生成手性高价碘策略成功地应用到烯烃类衍 生物 100 的不对称双胺化反应中, 得到一系列手性双环 产物 102, 产率为 $45 \% \sim 72 \%$, 最高 $e e$ 值可达 $86 \%$. 该 反应的部分产物在还原性条件下转化为游离的二胺(Eq. $37)$.

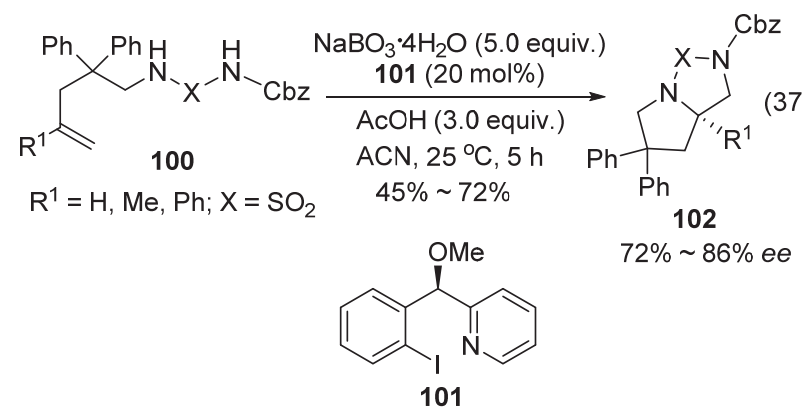

龚流柱课题组 ${ }^{[54]}$ 还报道了原位生成手性高价碘试 剂介导的一系列串联螺环化反应. $N, N$-二苯基丙二酰胺 类原料 103 在 $C 2$ 轴对称的手性碘代芳烃 104 催化氧化 作用下，活化四个 $\mathrm{C}-\mathrm{H}$ 键，发生分子内的 $\mathrm{C}-\mathrm{C}$ 键的氧 化偶联反应，以中等及以上收率和较高的 $e e$ 值得到具 有对映选择性的吲哚酮类化合物 $\mathbf{1 0 5}$, 最高 ee 值可达 $90 \%$ (Eq. 38). 2016 年, 杜云飞课题组 ${ }^{[55]}$ 使用 Gong 课题 组相同的手性碘代芳烃 104, 以 $m \mathrm{CPBA}$ 为氧化剂将一 系列 5-( $N$-甲基苯胺)-3,5-二羰基戊酸乙酯类底物 106 不 对称合成了螺吲哚呋喃类化合物 107, 该反应在室温下 进行，收率中等偏上，对应选择性好(Eq. 39). 对照试验 结果表明, 该不对称螺环化反应先进行的是碳氧键的偶 联再进行碳碳键的偶联, 碳碳键偶联反应是决定手性的 关键步骤.

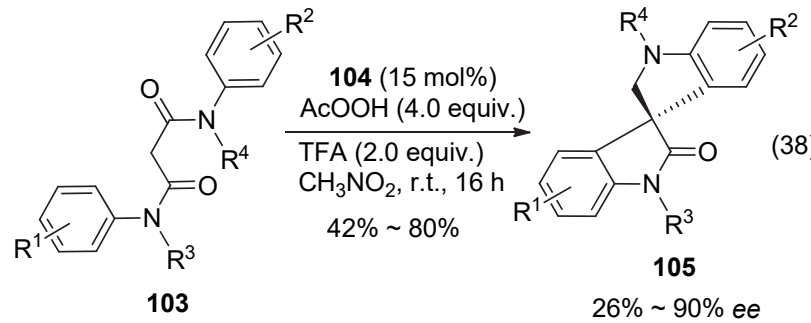

$\mathrm{R}^{1}=\mathrm{H}, 5-\mathrm{Me} ; \mathrm{R}^{2}=\mathrm{H}, 5-\mathrm{Ph}, 5-\mathrm{Cl}, 5-\mathrm{F}, 5-\mathrm{CO}_{2} \mathrm{Me}, 5-\mathrm{OMe}, 5-\mathrm{Me}$ $R^{3}=M e, B n$, etc.; $R^{4}=M e, B n$, etc.<smiles>[M]C(Oc1ccccc1OC(C)C(=O)N1CCCC1C(C)=O)C(=O)N1CCCC1OC(C)=O</smiles>

104

2018 年, Jacobsen 等 ${ }^{[56]}$ 发现烯丙基胺类化合物 108 在手性高价碘 109 和氧化剂 $m$ CPBA 作用下，与吡啶氢 氟酸可发生氧化环合生成 $\beta$-氟唑啶 110. 该反应需要在 低温下进行, 产率中等, 但立体选择性较高(Eq. 40). 
<smiles>[R10]C(=O)CC(=O)CC(=O)N([R])c1ccc([R20])cc1</smiles>

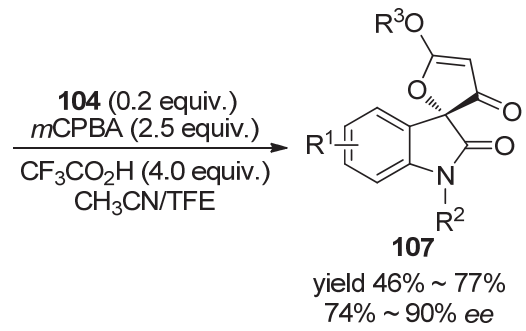

$\mathrm{R}^{1}=\mathrm{H}, 3-\mathrm{Me}, 3-\mathrm{OMe}, 3-\mathrm{COF}_{3}, 3-\mathrm{F}, 3-\mathrm{Cl}, 3-\mathrm{Br}, 5-\mathrm{Me}$

$\mathrm{R}^{2}=\mathrm{Me}, \mathrm{Et},{ }^{\mathrm{i}} \mathrm{Pr},{ }^{\mathrm{t}} \mathrm{Bu}, \mathrm{Bn}$

$\mathrm{R}^{3}=\mathrm{Me}, \mathrm{Et},{ }^{i} \mathrm{Pr},{ }^{n} \mathrm{Bu}$

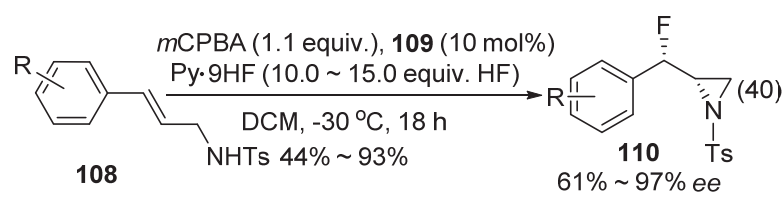

$\mathrm{R}=2-\mathrm{CF}_{3}, 3-\mathrm{NO}_{2}$, 3-OTf, 3- $\mathrm{Br}, 4-\mathrm{CF}_{3}, 4-\mathrm{SO}_{2} \mathrm{Me}, 4-\mathrm{NO}_{2}, 4-\mathrm{CN}, 4-\mathrm{OTf}$ etc.<smiles>COc1cc(OC(Cc2ccccc2)C(=O)OCc2ccccc2)c(I)c(OC(C(=O)OCc2ccccc2)C(=O)OCc2ccccc2)c1</smiles>

\section{5 结论和展望}

原位生成高价碘作为高价碘领域的一个新兴分支, 引发了许多化学家浓厚兴趣, 近十几年来围绕原位生成 高价碘介导的催化循环反应的研究成果层出不穷, 主要 包括原位生成三价碘、五价碘、手性高价碘在去芳构化、 环合反应和不对称催化等各种氧化反应中的应用.

原位生成策略采用催化量的芳基碘代物或者无机 碘化物代替等物质的量高价碘，这不仅避免了高价碘试 剂自身的不稳定性，也实现了原子经济性. 常用的氧化 剂反应生成的副产物多为常见的物质，如叔丁醇、甲酸、 水等, 安全可控且绿色环保. 该方法既保留了高价碘的 优点, 又符合绿色化学的理念, 已被广泛应用于 $\mathrm{C}-\mathrm{C}$, $\mathrm{C}-\mathrm{N}, \mathrm{C}-\mathrm{O}$ 键以及手性化合物的构建中. 但是原位生 成高价碘仍然面临很多挑战: (1)大部分原位生成反应需 要使用过氧化物作为氧化剂, 过氧化物易爆炸, 如何寻 找更多种类的氧化剂需要科研工作者进一步研究. (2)反 应过程不够高效, 部分反应需要时间过长, 尤其生成手 性化合物的过程. 还需深入研究反应条件, 开发高效氧 化剂和添加剂. (3)与传统高价碘相比, 原位生成高价碘 介导的反应类型偏简单, 在四元环、七元环、多元杂环和 复杂多环化合物的构建报道较少. 因此需要研究新的合 成方法制备不同类型的化合物, 更大发挥原位生成高价 碘在不同领域的应用.

随着高价碘化学、计算化学和应用化学的不断发展, 各种由原位生成的高价碘试剂介导的非金属条件下的
氧化反应有望不断涌现, 为具有药理活性的药物分子提 供重要的化合物来源.

\section{References}

[1] Musher, J. I. Angew. Chem. Int. Ed. 1969, 8, 54.

[2] (a) Sandin, R. B. Chem. Rev. 1943, 32, 249.

(b) Banks, D. F. Chem. Rev. 1966, 66, 243.

(c) Varvoglis, A. Tetrahedron 1997, 53, 1179.

(d) Tohma, H.; Kita, Y. Adv. Synth. Catal. 2004, 346, 111.

(e) Zhou, W.; Zhang, L.; Jiao, N. Angew. Chem., Int. Ed. 2009, 48, 7094.

(f) Mu, X.; Wu, T.; Wang, H.; Guo, Y.; Liu, G. J. Am. Chem. Soc. 2012, 134, 878 .

(g) Duan, X.; Yang, K.; Wang, Z.; Zhang, L.; Tong, Y.; Liu, H.; Du, K.; Tang, R. Chin. J. Org. Chem. 2015, 35, 2552 (in Chinese). (段希炎，杨坤，王志成，张璐，佟悦，刘浩哲，杜珂，唐榕泽， 有机化学, 2015, 35, 2552.)

[3] (a) Stang, P. J.; Zhdankin, V. V. Chem. Rev. 1996, 96, 1123. (b) Zhdankin, V. V.; Stang, P. J. Chem. Rev. 2002, 102, 2523.

(c) Moriarty, R. M. J. Org. Chem. 2005, 70, 2893.

(d) Zhdankin, V. V.; Stang, P. J. Chem. Rev. 2008, 108, 5299.

(e) Zhdankin, V. V. ARKIVOC 2009, $i, 1$.

(f) Kuepper, F. C.; Feiters, M. C.; Olofsson, B.; Kaiho, T.; Yanagida, S.; Zimmermann, M. B.; Carpenter, L. J.; Luther, G. W.; Lu, Z.; Jonsson, M.; Kloo, L. Angew. Chem., Int. Ed. 2011, 50, 11598.

(g) Charpentier, J.; Fruh, N.; Togni, A. Chem. Rev. 2015, 115, 650. (h) Yoshimura, A.; Zhdankin, V. V. Chem. Rev. 2016, 116, 3328.

(i) Duan, Y.; Jiang, S.; Han, Y.; Sun, B.; Zhang, C. Chin. J. Org. Chem. 2016, 36, 1973 (in Chinese).

(段亚南, 姜山, 韩永超, 孙博, 张弛, 有机化学, 2016, 36, 1973.)

(j) Zhang, X.; Cong, Y.; Lin, G.; Guo, X.; Cao, Y.; Lei, K.; Du, Y. Chin. J. Org. Chem. 2016, 36, 2513 (in Chinese).

(张翔, 从顷, 林光宇, 郭旭亮, 曹阳, 雷坤华, 杜云飞, 有机化 学, 2016, 36, 2513.)

(k) Han, Y.; Zhang, C. Tetrahedron Lett. 2018, 59, 3052.

(1) Cai, Q.; Ma, H. Acta Chim. Sinica 2019, 77, 213 (in Chinese).

(蔡倩, 马浩文, 化学学报, 2019, 77, 213.)

(m) Liu, Dan.; He J.; Zhang, C. Univ. Chem. 2019, 34, 1 (in Chinese).

(刘丹, 贺家豪, 张弛，大学化学， 2019, 34, 1.)

[4] Yang, L.; Zhang-Negrerie, D.; Zhao, K.; Du, Y. J. Org. Chem. 2016, $81,3372$.

[5] Yusubov, M. S.; Zhdankin, V. V. Curr. Org. Synth. 2012, 9, 247.

[6] Uyanik M, Ishihara K. ChemCatChem 2012, 4, 177.

[7] Trost, B. M. Science 1991, 254, 1471.

[8] Trost, B. M. Angew. Chem., Int. Ed. 1995, 34, 259.

[9] (a) Zheng, Z.; Zhang-Negrerie, D.; Du, Y.; Zhao, K. Sci. China, Chem. 2014, 57, 189.

(b) Zheng, Z. Ph.D. Dissertation, Tianjing University, Tianji, 2014 (in Chinese).

(郑子圣, 博士论文, 天津大学, 天津, 2014.)

(c) Singh, F. V.; Wirth, T. Chem.-Asian J. 2014, 9, 950.

(d) Parra, A.; Reboredo, S. Chem.-Eur. J. 2013, 19, 17244

[10] Uyanik, M.; Ishihara, K. Chem. Cat. Chem. 2012, 4, 177.

[11] Ochiai, M.; Takeuchi, Y.; Katayama, T.; Sueda, T.; Miyamoto, K. J. Am. Chem. Soc. 2005, 127, 12244.

[12] Yamamoto, Y.; Kawano, K.; Toy, P. H.; Togo, H. Tetrahedron 2007, 63, 4680.

[13] Dohi, T.; Maruyama, A.; Yoshimura, M.; Morimoto, K.; Tohma, H.; Kita, Y. Angew. Chem., Int. Ed. 2005, 44, 6193.

[14] Dohi, T.; Maruyama, A.; Minamitsuji, Y.; Takenaga, N.; Kita, Y. Chem. Commun. 2007, 1224.

[15] Zhu, C.; Liang, Y.; Hong, X.; Sun, H.; Sun, W.; Houk. K.; Shi, Z. J. Am. Chem. Soc. 2015, 137, 7564

[16] Liang, D.; Yu, W.; Nguyen, N.; Deschamps, J. R.; Imler, G. H.; Li, Y.; MacKerell, A. D.; Jiang, C.; Xue, F. J. Org. Chem. 2017, 82, 
3589.

[17] Ito, M.; Kubo, H.; Itani, I.; Morimoto, K.; Dohi, T.; Kita, Y. J. Am. Chem. Soc. 2013, 135, 14078.

[18] Miyamoto, K.; Sakai, Y.; Goda, S.; Ochiai, M. Chem. Commun. 2012, 48, 982

[19] Philips, B.; Frostick, F. C.; Starcher, P. S. J. Am. Chem. Soc. 1957, 79, 5982.

[20] Antonchick, A. P.; Samanta, R.; Kulikov, K.; Lategahn, J. Angew Chem., Int. Ed. 2011, 50, 8605.

[21] He, Y.; Huang, J.; Liang, D.; Liu, L.; Zhu, Q. Chem. Commun. 2013, 49, 7352 .

[22] Wang, X.; Gallardo-Donaire, J.; Martin, R. Angew. Chem., Int. Ed. 2014, 53, 11084.

[23] Shen, C.; Yang, M.; Xu, J.; Chen, C.; Zheng, K.; Shen, J.; Zhang, P. RSC Adv. 2017, 7, 49436.

[24] Braddock, D. C.; Cansell, G.; Hermitage, S. A. Chem. Commun. 2006, 2483.

[25] Fabry, D. C.; Stodulski, M.; Hoerner, S.; Gulder, T. Chem.-Eur. J. 2012, 18, 10834.

[26] Ulmer, A.; Stodulski, M.; Kohlhepp, S. V.; Patzelt, C.; Poethig, A.; Bettray, W.; Gulder, T. Chem.-Eur. J. 2015, 21, 1444.

[27] Patzelt, C.; Alexander, P.; Gulder, T. Org. Lett. 2016, 18, 3466.

[28] Stodulski, M.; Goetzinger, A.; Kohlhepp, S. V.; Gulder, T. Chem. Commun. 2014, 50, 3435.

[29] Liu, H.; Tan, C.-H. Tetrahedron Lett. 2007, 48, 8220.

[30] Alhalib, A.; Kamouka, S.; Moran, W. J. Org. Lett. 2015, 17, 1453.

[31] Morimoto, K.; Sakamoto, K.; Ohshika, T.; Dohi, T.; Kita. Y. Angew. Chem., Int. Ed. 2016, 55, 3652.

[32] Yoshimura, A.; Middleton, K. R.; Luedtke, M. W.; Zhu, C.; Zhdankin, V. V. J. Org. Chem. 2012, 77, 11399.

[33] Uyanik, M.; Suzuki, D.; Yasui, T.; Ishihara, K. Angew. Chem., Int. Ed. 2011, 50, 5331

[34] Ma, L.; Wang, X.; Yu, W.; Han, B. Chem. Commun. 2011, 47, 11333.

[35] Zhu, C.; Wei, Y. ChemSusChem 2011, 4, 1082.

[36] Guo, W.; Vallcorba, O.; Vallribera, A.; Shafir, A.; Pleixats, R.; Rius, J. Chem CatChem 2014, 6, 468.

[37] Zhu, C.; Zhang, Y.; Zhao, H.; Huang, S.; Zhang, M.; Su, W. Adv.
Synth. Catal. 2015, 357, 331.

[38] Duhamel, T.; Stein, C. J.; Martínez, C.; Reiher, M.; Muñiz. K. ACS Catal. 2018, 8,3918 .

[39] Thottumkara, A. P.; Bowsher, M. S.; Vinod, T. K. Org. Lett. 2005, 7,2933.

[40] Schulze, A.; Giannis, A. Synthesis 2006, 257.

[41] Uyanik, M.; Akakura, M.; Ishihara, K. J. Am. Chem. Soc. 2009, $131,251$.

[42] Uyanik, M.; Fukatsu, R.; Ishihara, K. Org. Lett. 2009, 11, 3470.

[43] Yusubov, M. S.; Zagulyaeva, A. A.; Zhdankin, V. V. Chem.-Eur. J. 2009, 15, 11091

[44] Richardson, R. D.; Page, T. K.; Altermann, S.; Paradine, S. M.; French, A. N.; Wirth, T. Synlett 2007, 538.

[45] Fenga, Y.; Huanga, R.; Hua, L.; Xiong, Y.; Coeffard, V. Synthesis 2016, 48, 2637.

[46] Pluta, R.; Krach, P. E.; Cavallo, L.; Falivene, L.; Rueping, M. ACS Catal. 2018, 8, 2582.

[47] Volp, K. A.; Harned, A. M. Chem. Commun. 2013, 49, 3001

[48] Dohi, T.; Maruyama, A.; Takenaga, N.; Senami, K.; Minamitsuji, Y.; Fujioka, H.; Caemmerer, S. B.; Kita, Y. Angew. Chem., Int. Ed. 2008, 47, 3787.

[49] Dohi, T.; Takenaga, N.; Nakae, T.; Toyoda, Y.; Yamasaki, M.; Shiro, M.; Fujioka, H.; Maruyama, A.; Kita, Y. J. Am. Chem. Soc. 2013, $135,4558$.

[50] Uyanik, M.; Yasui, T.; Ishihara, K. Angew. Chem., Int. Ed. 2010, 49, 2175.

[51] Dohi, T.; Sasa, H.; Miyazaki, K.; Fujitake, M.; Takenaga, N.; Kita, Y. J. Org. Chem. 2017, 82, 11954.

[52] Zhang, D.-Y.; Xu, L.; Wu, H.; Gong, L.-Z. Chem.-Eur. J. 2015, 21, 10314.

[53] Mizar, P.; Laverny, A.; Mohammad. E. S.; Farid, U.; Brown, M.; Malmedy, F.; Wirth, T. Chem. Eur. J. 2014, 20, 9910.

[54] Wu, H.; He, Y.-P.; Xu, L.; Zhang, D.-Y.; Gong, L.-Z. Angew. Chem., Int. Ed. 2014, 53, 3466.

[55] Cao, Y.; Zhang, X.; Lin, G.; Zhang-Negrerie, D.; Du, Y. Org. Lett. 2016, $18,5580$.

[56] Mennie, K. M.; Banik, S. M.; Reichert, E. C.; Jacobsen, E. N. $J$ Am. Chem. Soc. 2018, 140, 4797.

(Lu, Y. ) 